\title{
Definitive Chemoradiotherapy with Docetaxel, Cisplatin, and 5-Fluorouracil for Advanced Cervical Esophageal Cancer: A Medium-Term Outcome
}

\author{
Hiroshi Okamoto*, Yusuke Taniyama, Chiaki Sato, Toshiaki Fukutomi, Yohei \\ Ozawa, Ryohei Ando, Kozue Takahashi, Ryujiro Akaishi, Yuta Horie, Yasuharu \\ Shinozaki, Michiaki Unno, Takashi Kamei
}

\begin{abstract}
Background: Definitive chemoradiotherapy (dCRT) is widely considered as a treatment option for cervical esophageal squamous cell carcinoma (ESCC) toward preserving the larynx. We have reported favorable outcomes, including the treatment response rate and short-term survival of dCRT concomitant with docetaxel, cisplatin, and 5-fluorouracil (DCF-RT) for advanced cervical ESCC. The aim of this paper was to report the subsequent progress of the study. Methods: We assessed 18 patients with advanced (clinical stage II-IV, including T4b and/or M1 lymph node) cervical ESCC at our department who received DCF-RT as the first-line treatment between December 2010 and June 2020. Results: A total of 14 men and 4 women underwent the study regimen. The pretreatment clinical stage included stage II, stage III, stage IVA, and stage IVB cases (including 9 patients with T4b) [8 trachea and 2 thyroids] and 7 patients with the M1 lymph node. The complete response (CR) was achieved in 15 patients, stable disease in 2, and progressive disease in 1 . Of 15 patients with CR, 7 experienced recurrence, and 8 had continued CR. Frequent cases of grade $\geq 3$ adverse effects included leucopenia, neutropenia, febrile neutropenia, and pharyngeal pain. The 3-year overall survival rate, disease-free survival rate, and disease-specific survival rate were $44.2 \%, 47.7 \%$, and 48.6\%, respectively. Conclusion: DCF-RT for advanced cervical esophageal cancer could achieve a favorable prognosis with larynx preservation. Further observations are warranted to establish the long-term prognosis, late complications of radiotherapy, and the significance of salvage surgery.
\end{abstract}

Keywords: Cervical esophageal cancer- squamous cell carcinoma- definitive chemoradiotherapy- docetaxel-cisplatin

Asian Pac J Cancer Prev, 23 (2), 495-499

\section{Introduction}

Esophageal cancer is one of the common types of cancer across the world, among which the prevalence of cervical esophageal carcinoma $(4.8 \%)$ is lesser than that of thoracic esophageal carcinoma (Watanabe et al., 2021). The use of definitive chemoradiotherapy (dCRT) is the preferred choice of treatment for cervical esophageal squamous cell carcinoma (ESCC) that has been shown to preserve the progression of disease in the larynx. In our previous study, the treatment response rate and the short-term survival of dCRT concomitant with docetaxel, cisplatin, and 5-fluorouracil (DCF-RT) for advanced cervical ESCC were evaluated for the first time (Okamoto et al., 2018). Herein, we report the subsequent progress of our previous study using DCF-RT for advanced cervical ESCC.

\section{Materials and Methods}

In this study, 18 patients with advanced (clinical stage II-IV, including T4b and/or M1 lymph node) cervical ESCC who received DCF-RT as the first-line treatment between December 2010 and July 2020 at our hospital were examined and considered for the study. The protocols of this study and the regimen of DCF-RT given to the patients were as per the literature (Okamoto et al., 2018). The adopted protocol of chemotherapy comprised of 2 cycles, each of docetaxel (i.v., $60 \mathrm{mg} / \mathrm{m}^{2}$ ) and cisplatin $\left(60 \mathrm{mg} / \mathrm{m}^{2}\right)$ infusion on day 2 , followed by continuous infusion of 5-fluorouracil (5-FU; $1000 \mathrm{mg}$ /day) from days $1-5$, every 4 weeks. In concurrent radiotherapy, the target volume is comprised of the primary tumor, recurrent nerve, supraclavicular, and deep cervical lymph node regions. After administering a dose of $40 \mathrm{~Gy}$, an extra 
boost of radiation was administered to the primary tumor and clinically metastatic lymph nodes of the patients. However, the total dose of irradiation was 60-70 Gy, with a fractionation dose of 2 Gy.

The clinical stage was diagnosed according to the 8th edition of the TNM Classification of the Union for International Cancer Control (James et al., 2017) and the adverse effects were defined according to the Common Terminology Criteria for Adverse Events version 4.0 (CTCAE 2010). The response evaluation was performed by esophagogastroduodenoscopy (EGD) with biopsy, enhanced computed tomography (CT), and a 2-[fluorine-18] fluoro-2-deoxy-D-glucose positron emission tomography (FDG-PET); all these examinations were performed within 2 months after the completion of treatment. The survival time was determined from day 1 of treatment until death (overall survival [OS] or diseasespecific survival [DSS])/recurrence (disease-free survival [DFS]) or the last follow-up examination and the survival curve was analyzed according to the Kaplan-Meier method. The frequency of the outpatient follow-up was scheduled once in every 3 months until 2 years from day 1 of the treatment and once every 4-6 months afterward, either through a blood test, EGD with biopsy, CT, and FDG-PET, based on the suitability

This study was approved by the ethical committee of our institute (accession number: 2017-4-064), and we

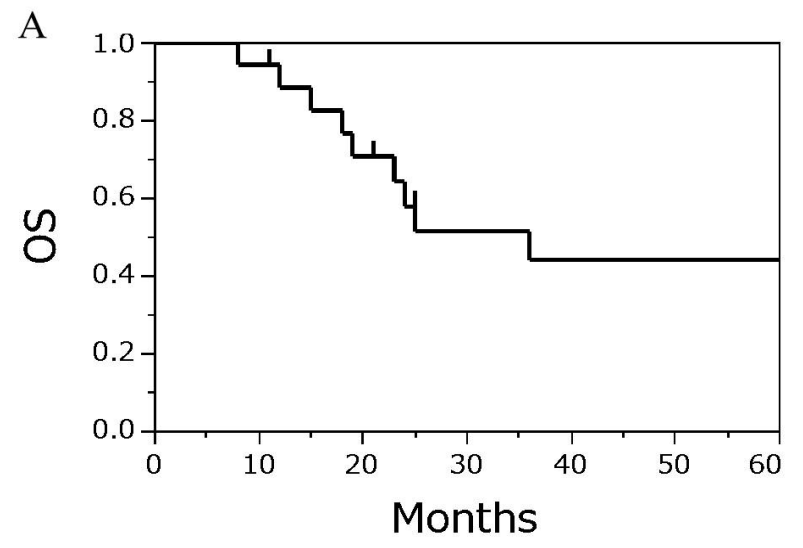

obtained informed consent from all patients. We conducted this study following the Declaration of Helsinki (1975).

\section{Results}

The patients' characteristics and clinical outcomes in this study have been summarized (Table 1), and the cohort comprised of 14 men and 4 women (median age $=66$ years; age range: 54-76 years). There were 7 patients with $\mathrm{T} 4 \mathrm{~b}$ (tracheae), 1 patient with T4b (thyroid), and 1 patient with T4b (trachea and thyroid). The clinical stage of cancer in patients included stage II $(n=1)$, stage III $(n=5)$, stage $\operatorname{IVA}(\mathrm{n}=5)$, and stage IVB $(\mathrm{n}=7$ with M1 supraclavicular lymph node). Intensity-modulated radiotherapy (IMRT) was used for the recent 6 patients. The median observation period for all the patients was 24.5 (8-125) months, while that for the 8 survived patients was 85 (11-125) months.

All the studied patients had completed their DCF-RT schedule, although, in 4 patients $(22 \%)$, the drug dose used in the second cycle of chemotherapy was reduced due to the adverse effects. The adverse effects observed during the study are listed in Table 2. The adverse effects of $\geq$ the grade 3 score included leucopenia (94\%), neutropenia (89\%), febrile neutropenia $(61 \%)$, and pharyngeal pain (44\%). It is quintessential to mention that no treatment-related mortality was recorded in the study. During the treatment, it was encountered that

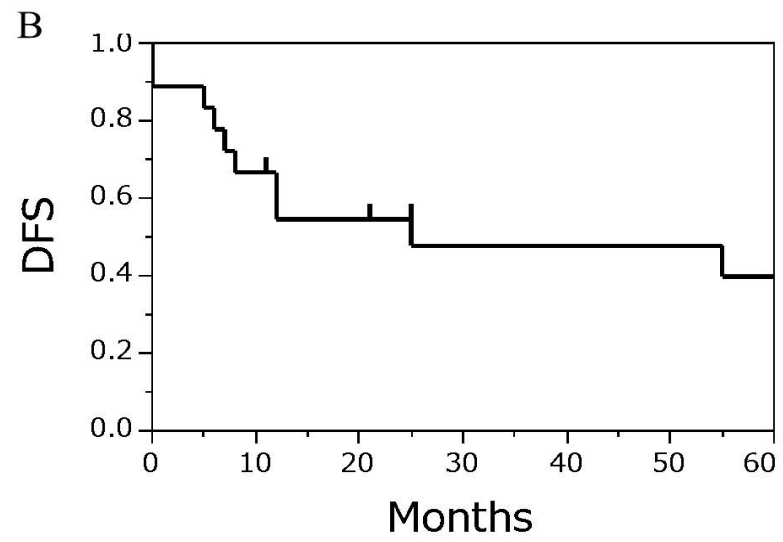

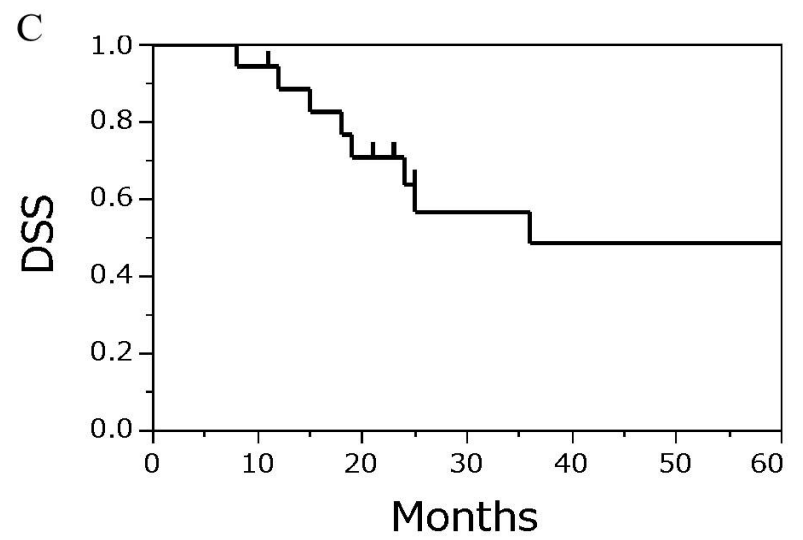

Figure 1. The Survival Curve by the Kaplan-Meier Method. A, The overall survival curve. The overall survival rate at 2,3 , and 5 years was $58.0 \%, 44.2 \%$, and $39.8 \%$, respectively; B, The disease-free survival curve. The DFS rate at 2,3 , and 5 years was $54.6 \%, 47.7 \%$, and $38.6 \%$, respectively; $\mathrm{C}$, The disease-specific survival curve. The disease-specific survival rate at 2,3 , and 5 years was $63.8 \%, 48.6 \%$, and $48.6 \%$, respectively. 
Table 1. Patient's Characteristics

\begin{tabular}{|c|c|}
\hline Variables & Total $n=18(\%)$ \\
\hline \multicolumn{2}{|l|}{ Age (years) } \\
\hline Median & 66 \\
\hline (Range) & $(43-79)$ \\
\hline \multicolumn{2}{|l|}{ Gender } \\
\hline Male & $14(77.8)$ \\
\hline Female & $4(22.2)$ \\
\hline \multicolumn{2}{|l|}{$\mathrm{cT}$} \\
\hline cT3 & $9(50.0)$ \\
\hline cT4a & $9(50.0)$ \\
\hline \multicolumn{2}{|l|}{$\mathrm{cN}$} \\
\hline cNO & $3(16.7)$ \\
\hline $\mathrm{cN} 1$ & $9(50.0)$ \\
\hline $\mathrm{cN} 2$ & $5(27.8)$ \\
\hline $\mathrm{cN} 3$ & $1(5.6)$ \\
\hline \multicolumn{2}{|l|}{$\mathrm{cM}$} \\
\hline $\mathrm{cM} 0$ & $11(61.1)$ \\
\hline cM1 & 7 (38.9) \\
\hline \multicolumn{2}{|l|}{ cStage } \\
\hline cStage II & $1(5.6)$ \\
\hline cStage III & $5(27.8)$ \\
\hline cStage IVA & $5(27.8)$ \\
\hline cStage IVB & $7(38.9)$ \\
\hline \multicolumn{2}{|c|}{ Radiation dose (Gy) } \\
\hline 60 & $12(66.7)$ \\
\hline 62.4 & $1(5.6)$ \\
\hline 66 & $1(5.6)$ \\
\hline 70 & $4(22.2)$ \\
\hline \multicolumn{2}{|c|}{ Additional chemotherapy } \\
\hline Done & $1(5.6)$ \\
\hline None & $17(94.4)$ \\
\hline
\end{tabular}

the oral intake was difficult and hence 6 patients had to undergo percutaneous endoscopic gastrostomy (PEG), 1 patient (after gastrectomy) jejunostomy, and 3 patients nasogastric tube.

The complete response (CR) was successfully achieved in 15 patients, whereas stable disease was recorded in 2 patients and progressive disease in 1 patient. The data revealed that the $\mathrm{CR}$ rate was equal to the response rate $(83.3 \%)$. Among the $\mathrm{CR}$ in 15 patients, 7 had experienced the recurrence of disease and 8 had continued CR. Two patients with persistent or recurrent disease underwent salvage surgery. At the moment, no delayed adverse events due to radiotherapy were observed. The observations of the study in terms of survival reflect that the OS rate at 2, 3, and 5 years was 58.0, 44.2, and $39.8 \%$, respectively, and the DFS rate at 2,3 , and 5 years was $54.6,47.7$, and $38.6 \%$, respectively. The DSS rate at 2,3 , and 5 years was $63.8,48.6$, and $48.6 \%$, respectively (Figures 1A, 1B, and 1C).
Table 2. Adverse Events (CTCAE version 4.0)

\begin{tabular}{lccccc}
\hline & G1 & G2 & G3 & G4 & G3/G4 (\%) \\
\hline Leukopenia & 0 & 1 & 10 & 7 & 94 \\
Neutropenia & 0 & 1 & 5 & 11 & 89 \\
Febrile neutropenia & - & - & 11 & 0 & 61 \\
Anemia & 8 & 6 & 3 & 0 & 17 \\
Thrombocytopenia & 6 & 3 & 1 & 0 & 9 \\
Increased creatinine & 1 & 1 & 0 & 0 & 0 \\
Nausea/anorexia & 0 & 3 & 5 & - & 28 \\
Diarrhea & 2 & 1 & 1 & 0 & 9 \\
Alopecia & 4 & 0 & - & - & - \\
Pharyngeal pain & 2 & 3 & 8 & - & 44 \\
Radiation dermatitis & 2 & 6 & 0 & 0 & 0 \\
Arrhythmia (PSVT) & 0 & 2 & 0 & 0 & 0 \\
Urticaria & 1 & 0 & 0 & 0 & 0 \\
Oral mucositis & 2 & 1 & 2 & 0 & 11 \\
\hline
\end{tabular}

PSVT, paroxysmal supraventricular tachycardia

\section{Discussion}

Cervical esophagus cancer is particularly difficult to observe in detail and therefore early detection of disease recurrence is required. The detailed observations were recorded through EGD along with a repeated biopsy and a close follow-up. Although some time has passed since our previous report and the number of cases has increased since then, the response rate continues to remain high despite the quite advanced cases. It was observed during the study that the condition in $46.7 \%$ of the CR patients had recurred and that the OS after recurrence was severe with a median of 11 (7-19) months. The CR and recurrence were however difficult to measure, because the tumor cell nests often exists deeply within the esophageal wall (Fujishima et al., 2018).

One of the most troublesome problems after dCRT is stenosis or obstruction at the tumor site in the cervical esophagus because it is anatomically obstructed with the progression of the disease. The patients in this study had gradually started eating to some extent; however, 4 patients (22\%) received nourishment using PEG after dCRT. Another 3 patients underwent endoscopic dilatation, 2 of whom underwent endoscopic incision with an electric knife. In the case of complete obstruction, the secretion of saliva in patients (out of the mouth) was observed to impair the quality of life. When cervical esophagus is completely occluded, it is necessary to secure a route for treatment, such as through endoscopic dilatation and incision by using a tube. In such case, PEG was used to pass the guidewire. When esophageal cancer recurs and ectopically and endoscopic treatments are necessary, difficulty is encountered in the passage during endoscopic treatment due to stenosis. During this situation in our study, the patient underwent endoscopic photodynamic therapy via PEG. In these patients, retaining the PEG for subsequent treatment is advisable even after the patient is capable of eating as per the OS. 
dCRT of cervical ESCC is often selected for the preservation of patient larynx and DCF-RT seems to achieve a high response rate; therefore, the concurrent chemotherapy is deemed beneficial for prognosis. The5-fluorouracil and platinum-based chemotherapy (FP-RT) has been used for dCRT of the cervical esophagus in several reports. These previous studies suggest that the OS rate after 5-year was 21\%-55\% (Burmeister et al., 2000; Yamanda et al., 2006; Uno et al., 2007; Gkika et al., 2014; Zhang et al., 2015; Zenda et al., 2016; Ito et al., 2017; Li et al., 2017). As evident from this study, the degree of disease progression in patients was far advanced and the survival rate was also satisfactory. The literature supports the priority of DCF-RT over FP-RT for CR rate (36.7\% vs 3.7\%) and 3-year OS (32.8\% vs $8.5 \%$ ) of patients with unresectable locally advanced thoracic ESCC (Sakai et al., 2021). Although Sakai et al. reported that DCF-RT was effective in treating thoracic ESCC, DCF-RT can also be expected to have a good prognostic effect on cervical ESCC. Although a randomized clinical trial is necessary to clarify whether DCF-RT can achieve a better prognosis of patients with cervical ESCC than FP-RT, which is otherwise difficult because of the low incidence of cervical ESCC. If you do not care about larynx preservation, it is possible to use DCF as an induction, and conversion surgery is required if it becomes possible to resect. In a similar context, the ongoing nationwide phase 3 clinical trial (JCOG1510) may be helpful to select a treatment regime that is better for prognosis that is the DCF-RT or conversion surgery. The outcomes of this clinical trial are expected to reveal the superiority of induction by DCF followed by conversion surgery (when it gets to be resectable after $\mathrm{DCF}$ ) or dCRT (when it is still unresectable) over dCRT alone for locally advanced unresectable thoracic ESCC. The results of this study are presently awaited.

Furthermore, 2 patients underwent salvage surgery during our study: 1 patient with the persistent disease had total esophagostomy with larynx preservation but died after 23 months of treatment initiation (17 months after the surgery) without recurrence due to lung cancer. The other patient with recurrent disease underwent total laryngopharyngoesophagectomy and died of primary disease 19 months after the initiation of treatment (6 months after the surgery). A study by Takebayashi (2017) reported that the 3-year recurrence-free survival rate and the OS rate after salvage laryngopharyngoesophagectomy for residual or recurrent tumors of cervical esophageal cancer was $49.87 \%$ and $70.13 \%$. They also concluded that salvage surgery can offer the exclusive chance of prolonged survival. However, there is a risk of tracheal ischemia and thus salvage surgery must be taken performed carefully. Although the number of cases is small in this study, it may be an effective treatment option.

Irradiation methods are advancing with time, and intensity-modulated radiotherapy (IMRT) and volumetricmodulated arc therapy for cervical ESCC have been reported and demonstrated as a favorable one that can ultimately lead to the reduction of radiation injury (Ito et al., 2017; Cao et al., 2016; Yanh et al., 2017; Zhao et al., 2017; Chen et al., 2019). Through our continuous efforts since 2018, we have introduced DCF-RT with IMRT for cervical ESCC, without any experience of late complications to date; however, further extensive observations are needed for validating the safety and efficacy of this approach.

In conclusion, the use of DCF-RT for advanced cervical ESCC could achieve a favorable prognosis with larynx preservation also in the medium term. Further detailed observations and case accumulation are needed for the long-term prognosis, late complications of radiotherapy, and the significance of salvage surgery.

\section{Author Contribution Statement}

Okamoto $\mathrm{H}$ is the main author of this article; Sato C, Fukutomi T, Ozawa Y, Ando R, Takahashi K, Akaishi R, Horie $\mathrm{Y}$, and Shinozaki Y contributed to collection and assembly of data and prepared the manuscript; Taniyama Y, Unno M, and Kamei T reviewed the manuscript. All authors have read and approved the final manuscript.

\section{Acknowledgments}

The authors thank Enago (www.enago.jp) for the English language review.

\section{Funding Statement}

This work has been supported by KAKENHI grants from the Ministry of Education, Culture, Sports, Science, and Technology of Japan to Okamoto H (18K16296).

\section{Ethics committee}

This study was approved by the ethical committee of our institute (accession number: 2017-4-064).

\section{Handling of the ethical issue}

We obtained informed consent from all patients. We conducted this study as per the Declaration of Helsinki (1975).

\section{Availability of data}

The dataset is available from the corresponding author on reasonable request.

\section{Statement conflict of interest}

We have no conflict of interest to disclose.

\section{References}

Burmeister BH, Dickie G, Smithers BM, Hodge R, Morton K (2000). Thirty-four patients with carcinoma of the cervical esophagus treated with chemoradiation therapy. Arch Otolaryngol Head Neck Surg, 126, 205-8.

Chen F, Li J, Ai N, et al (2019). Influence of 3D-CRT and conformal IMRT on thyroid function of patients with cervical and upper thoracic esophageal cancer and comparison of clinical efficacy. Oncol Lett, 17, 3432-8.

Cao CN, Luo JW, Gao L, et al (2016). Intensity-modulated radiotherapy for cervical esophageal squamous cell carcinoma: clinical outcomes and patterns of failure. Eur Arch Otorhinolaryngol, 273, 741-7. 
Fujishima F, Taniyama Y, Nakamura Y, et al (2018). Residual carcinoma cells after chemoradiotherapy for esophageal squamous cell carcinoma patients: striving toward appropriate judgment of biopsy. Dis Esophagus, 31, 1-6.

Gkika E, Gauler T, Eberhardt W, et al (2014). Long-term results of definitive radiochemotherapy in locally advanced cancers of the cervical esophagus. Dis Esophagus, 27, 678-84.

Ito M, Kodaira T, Tachibana H, et al (2017). Clinical results of definitive chemoradiotherapy for cervical esophageal cancer: comparison of failure pattern and toxicities between intensity-modulated radiotherapy and 3-dimensional conformal radiotherapy. Head Neck, 39, 2406-15.

James DB, Gospodarowicz MK, Wittekind C (2018). TNM classification of malignant tumors, 8th ed., Wiley-Blackwell, pp 57-62.

Li HX, Liu J, Cheng Y, et al (2017). Concurrent chemoradiotherapy for cervical esophageal squamous cell carcinoma: treatment results from a prospective observational study. Dis Esophagus, 31, 1-6.

National Cancer Institute Common Terminology Criteria for Adverse Events (CTCAE), Version 4.0. Available from: http://ctep.cancer.gov/protocolDevelopment/electronic applications/ctc.htm. [Last accessed on 6 June 2021].

Okamoto H, Taniyama Y, Sakurai T, et al (2018). Definitive chemoradiotherapy with docetaxel, cisplatin, and 5 -fluorouracil (DCF-R) for advanced cervical esophageal cancer. Esophagus, 15, 281-5.

Sakai M, Sohda M, Saito H, et al (2021). Concurrent chemoradiotherapy with docetaxel, cisplatin, and 5-fluorouracil (DCF-RT) vs. cisplatin and 5-fluorouracil (CF-RT) for patients with unresectable locally advanced esophageal cancer in a real-world clinical setting. Anticancer Res, 41, 2141-5.

Takebayashi K, Tsubosa Y, Kamijo T, et al (2017). Comparison of salvage total pharyngolaryngectomy and cervical esophagectomy between hypopharyngeal cancer and cervical esophageal cancer. Ann Surg Oncol, 24, 778-84.

Uno T, Isobe K, Kawakami H, et al (2007). Concurrent chemoradiation for patients with squamous cell carcinoma of the cervical esophagus. Dis Esophagus, 20, 12-8.

Watanabe M, Tachimori Y, Oyama T, et al (2021). Comprehensive registry of esophageal cancer in Japan, 2013. Esophagus, 18, $1-24$.

Yamada K, Murakami M, Okamoto Y, et al (2006). Treatment results of radiotherapy for carcinoma of the cervical esophagus. Acta Oncol, 45, 1120-5.

Yang H, Feng C, Cai BN,Yang J, Liu HX, Ma L (2017). Comparison of three-dimensional conformal radiation therapy, intensity-modulated radiation therapy, and volumetric-modulated arc therapy in the treatment of cervical esophageal carcinoma. Dis Esophagus, 30, 1-8.

Zenda S, Kojima T, Kato K, et al (2016). Multicenter phase 2 study of cisplatin and 5-fluorouracil with concurrent radiation therapy as an organ preservation approach in patients with squamous cell carcinoma of the cervical esophagus. Int J Radiat Oncol Biol Phys, 96, 976-84.

Zhang P, Xi M, Zhao L, et al (2015). Clinical efficacy and failure pattern in patients with cervical esophageal cancer treated with definitive chemoradiotherapy. Radiother Oncol, 116, 257-61.

Zhao L, Zhou Y, Mu Y, et al (2017). Patterns of failure and clinical outcomes of definitive radiotherapy for cervical esophageal cancer. Oncotarget, 8, 21852-60.

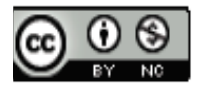

This work is licensed under a Creative Commons AttributionNon Commercial 4.0 International License. 